\title{
Microwaves and nanoparticles: from synthesis to imaging
}

Kenith E. Meissner, Ravish Majithiaa, R. A. Brown, Lihong V. Wang, T. G. G. Maffeis

Kenith E. Meissner, Ravish Majithiaa, R. A. Brown, Lihong V. Wang, T. G. G. Maffeis, "Microwaves and nanoparticles: from synthesis to imaging," Proc. SPIE 7909, Colloidal Quantum Dots/Nanocrystals for Biomedical Applications VI, 79091E (11 February 2011); doi: 10.1117/12.891242

SPIE. Event: SPIE BiOS, 2011, San Francisco, California, United States 


\title{
Microwaves and Nanoparticles: From Synthesis to Imaging
}

\author{
Kenith E. Meissner ${ }^{*}$,b ${ }^{\text {, Ravish Majithia }}{ }^{b}$, R. A. Brown ${ }^{c}$, Lihong V. Wang ${ }^{\mathrm{d}}$ and T. G. G. Maffeis ${ }^{\mathrm{c}}$ \\ ${ }^{a}$ Dept. of Biomedical Engineering, Texas A\&M Univ., College Station, TX 77843 \\ ${ }^{\mathrm{b}}$ Materials Science and Engineering Program, Texas A\&M Univ., College Station, TX 77843 \\ ${ }^{\mathrm{c}}$ College of Engineering, Swansea Univ., Swansea, Wales, UK \\ ${ }^{\mathrm{d}}$ Dept. of Biomedical Engineering, Washington Univ. in St. Louis, St. Louis, MO 63130-4899
}

\begin{abstract}
We investigate the use of energy delivery using microwave radiation for both synthesis of nanoparticles as well as a hybrid imaging technique known as thermoacoustic tomography (TAT). In each instance, the absorption of microwave radiation is converted into heat. In the case of nanoparticle synthesis, water is used as the solvent and heated to induce synthesis of the nanostructures. For this aqueous synthesis technique, we demonstrate the use of both pulsed and continuous wave (CW) microwave systems operating at $2.45 \mathrm{GHz}$. In this report, we concentrate on $\mathrm{ZnO}$ nanostructures including nanorods, nanowire arrays and nanobelts. These are compared with nanowire arrays and nanobelts grown by vapor transport through both electron microscopy and photo-excited luminescence. We also review the use of iron oxide $\left(\mathrm{Fe}_{3} \mathrm{O}_{4}\right)$ nanoparticles as contrast agents in TAT as previously reported. Here, we measured the properties of the colloidal nanoparticles in the microwave regime and compared the absorption with the TAT signal produced by our thermoacoustic imaging system at $3 \mathrm{GHz}$. The nanoparticles directly absorb the microwave radiation and produce a thermo-acoustic signal. The results from nanoparticles are compared to the signal produced by deionized water. The results demonstrate that microwaves represent an efficient method for the delivery of energy for both synthesis and biomedical imaging.
\end{abstract}

Keywords: Nanoparticles, quantum dots, nanowires, thermoacoustic tomography

\section{INTRODUCTION}

Microwave-assisted synthesis of nanoparticles has experienced rapid growth in the last 10 years. Conventional heating methods, such as convective and conductive heating, are primarily used for high temperature initiated nanoparticle synthesis. Common heating apparatuses include heated oil baths, heating mantles and sand baths. The transfer of energy from heat source, across the reaction vessel to the solvent and finally to the reactant molecules is inefficient and results in acute thermal gradients through the bulk solution causing non-uniform heating conditions. ${ }^{1}$ Also, this indirect heat transfer is a time consuming process that provides limited control over the reaction parameters. Control over reaction parameters such as nucleation time and reaction temperature is a key factor in nanostructure growth. Following the development and use of microwave ovens for heating in domestic kitchen applications, microwave technology has been applied as an alternative heating source for organic synthesis ${ }^{2,3}$ as well as nanoparticle synthesis ${ }^{4,5}$. Microwaves can provide sufficient energy to induce chemical transformations in a more efficient manner compared to conventional heating methods. Energy is transferred rapidly and directly through the reaction vessel to the microwave absorbing material (either solvent or chemical species) which subsequently results in localized superheating of the reactant molecules.

${ }^{*}$ To whom all correspondence should be addressed. Telephone: 979-458-0180; Fax: 979-845-4450;

Email: kmeissner@tamu.edu

Colloidal Quantum Dots/Nanocrystals for Biomedical Applications VI, edited by Wolfgang J. Parak, Kenji Yamamoto, Marek Osinski, Proc. of SPIE Vol. 7909, 79091E · C 2011 SPIE · CCC code: 1605-7422/11/\$18 · doi: 10.1117/12.891242 
Microwave-induced thermoacoustic tomography (TAT) is a hybrid imaging technique that combines the contrast provided by microwave energy with the spatial resolution of thermoacoustic waves. ${ }^{6,7}$ Generally, this technique relies on the endogenous contrast provided primarily by water content and ionic concentration in tissue. While TAT provides excellent imaging when there are differences in these endogenous agents, the development of exogenous contrast agents would revolutionize TAT. Since water content and ionic concentration limit the ability of microwaves to penetrate tissue, the development of exogenous contrast agents would serve to decouple contrast from penetration depth and enable applications where the endogenous contrast is insufficient. As previously reported, iron oxide $\left(\mathrm{Fe}_{3} \mathrm{O}_{4}\right)$ nanoparticles ${ }^{8,9}$ and carbon nanotubes ${ }^{10}$ have been investigated as contrast agents in the microwave (300 $\mathrm{MHz}-300$ $\mathrm{GHz}$ ) spectral region. Iron oxide nanoparticles were selected due to known absorption in the microwave region and their resulting use as contrast agents in MRI imaging. Here, we show that the iron oxide nanoparticle colloid provides an efficient TAT contrast agent compared to deionized water at $3 \mathrm{GHz}$. Finally, a reconstructed two-dimensional TAT image of iron oxide colloids in a tissue phantom is shown to demonstrate the efficacy of the technique.

\section{RESULTS AND DISCUSSION}

\subsection{Microwave Synthesis of Nanoparticles}

Typical kitchen microwave ovens have recently been used to fabricate $\mathrm{ZnO}$ nanowire arrays in a simple, aqueous-based technique. ${ }^{5}$ In this case, pulsed microwave energy at $2.45 \mathrm{GHz}$ is delivered to the sample without temperature control. We have synthesized nanowire arrays using this technque. In additional to a standard kitchen microwave oven, we have employed a commercially available microwave reactor specially designed for chemical synthesis (Discover ${ }^{\circledR}$ BenchMate, CEM Corporation, NC). This system is equipped with single mode microwave cavity at $2.45 \mathrm{GHz}$ with maximum power rating of $300 \mathrm{~W}$. It has a built in magnetic stirrer mechanism and an infra red (IR) probe for accurate, remote temperature measurement. This instrument provides the capability to dynamically control the synthesis temperature profile using bundled computer software.

\subsubsection{Colloidal ZnO Nanorods}

Colloidal $\mathrm{ZnO}$ nanorods were synthesized by mixing zinc nitrate hexahydrate $\left(\mathrm{Zn}\left(\mathrm{NO}_{3}\right)_{2} \cdot 6 \mathrm{H}_{2} \mathrm{O}\right)(99 \%$ purity, SigmaAldrich) and Hexamethylenetetraamine (HMT, Sigma Aldrich) $\left(\mathrm{C}_{6} \mathrm{H}_{12} \mathrm{~N}_{4}\right)$ solutions in a 2:1 molar ratio. Solutions of zinc nitrate at two different concentrations, namely at $100 \mathrm{mM}$ and $25 \mathrm{mM}$ were used. A $2 \mathrm{ml}$ mixture of the precursors was heated to $170 \mathrm{C}$ under a pressure of $\sim 110 \mathrm{psi}$ at $\sim 85 \mathrm{~W}$ of microwave power for a period of 20 minutes. Rods were dropcasted onto a glass microslide for SEM imaging. ZnO rods generated at $100 \mathrm{mM}$ Zinc nitrate concentration are $\sim 1.8$ $\mu \mathrm{m}$ long and $\sim 0.4 \mu \mathrm{m}$ wide (Figure 1). Lower concentration of $25 \mathrm{mM}$ Zinc nitrate generates longer rods $(\sim 2.4 \mu \mathrm{m})$ having similar widths $(\sim 0.4 \mu \mathrm{m})$ (Figure 1$)$.
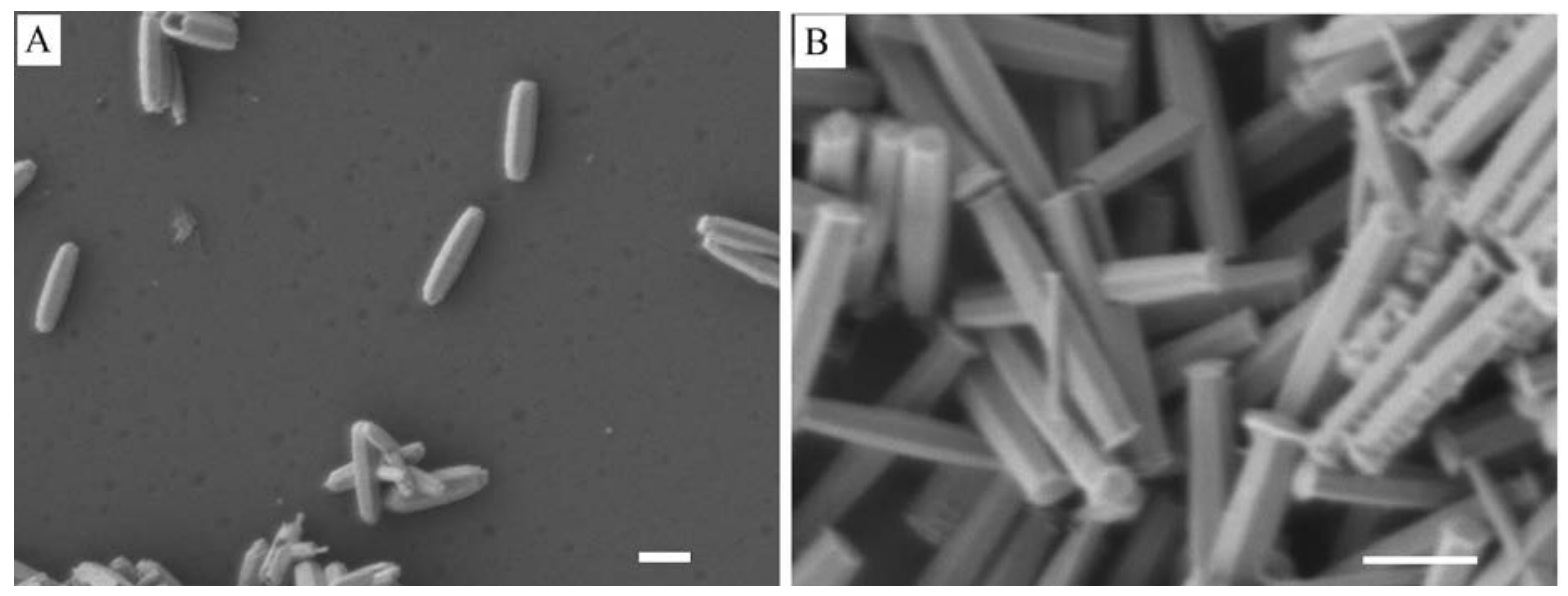

Figure 1: SEM images of colloidal ZnO rods synthesized by using microwaves at 2:1 molar ratio of zinc nitrate and HMTA at (A) $100 \mathrm{mM}$ zinc nitrate concentration and (B) $25 \mathrm{mM}$ zinc concentration. Scale bars $=1 \mu \mathrm{m}$. 


\subsubsection{ZnO Nanowire Arrays}

Arrays of $\mathrm{ZnO}$ nanorods were grown by the hydrothermal technique in a water bath and with a microwave system on glass cover slips and silicon wafers, respectively. In both experiments, a seed layer of $\mathrm{ZnO}$ was formed on the surface by dropping $25 \mu \mathrm{l}$ of $0.1 \mathrm{M}$ Zinc Acetate Dihydrate in ethanol on the surface, spinning at $3000 \mathrm{rpm}$ for 30 seconds and drying. This process was repeated twice and then the substrate baked at $350 \mathrm{C}$ for 15 minutes. After washing, sonicating and drying the samples, the substrates were suspended in reaction vessels and covered with an aqueous solution containing $25 \mathrm{mM}$ of both Hexamethylenetetramine (HMTA) and Zinc Nitrate Hexahydrate. In the case of water bath growth, the system was held at $90 \mathrm{C}$ for 9 hours. As shown in Figure 2(b), the growth produced ZnO nanowires with typical diameters of 35-70 $\mathrm{nm}$ and length of approximately $3 \mu \mathrm{m}$. In the case of the microwave growth, the system was held at $150 \mathrm{C}$ in a pressurized vessel for 5 minutes. As shown in Figure 2(a), the growth produced $\mathrm{ZnO}$ nanowires with similar diameters and lengths on the order of 100-200 nm. Thus, growth rate has been greatly accelerated by use of the microwave.
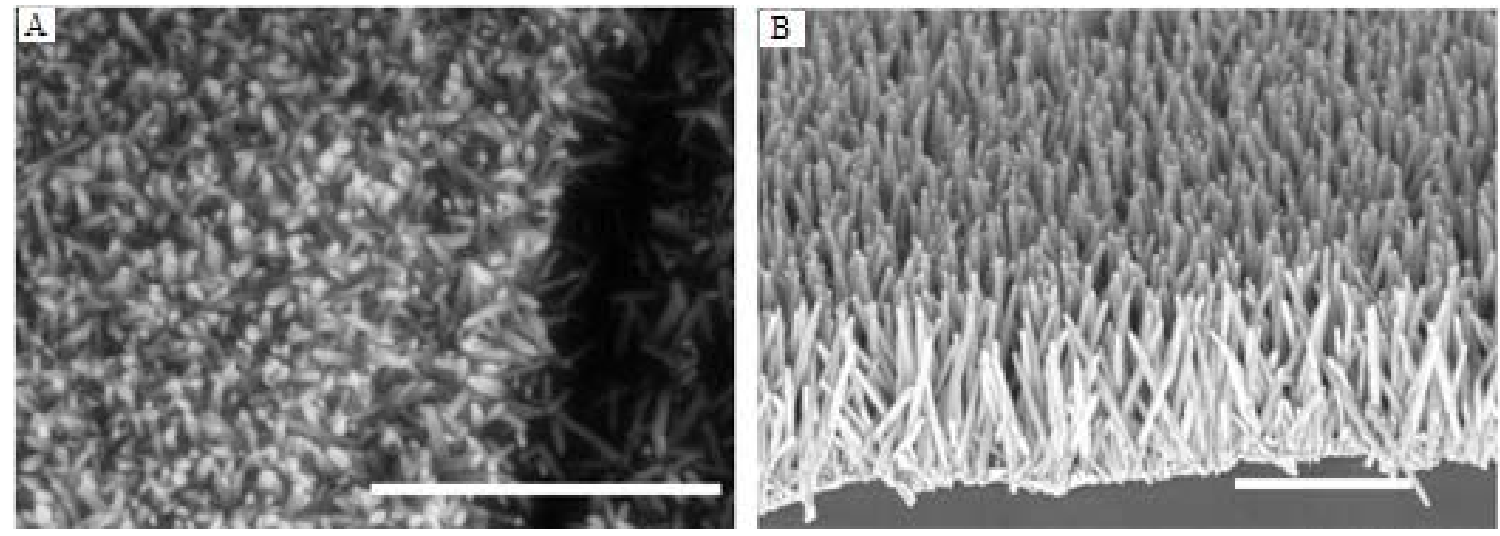

Figure 2: $\mathrm{ZnO}$ nanowire arrays grown by the hydrothermal technique. (A) Growth using a microwave system at 170 $\mathrm{C}$ for 5 minutes. (B) Growth using a water bath at $90 \mathrm{C}$ for 9 hours. Scale bars $=1 \mu \mathrm{m}$.

\subsection{Nanoparticles as Contrast Agents in Microwave-based Thermoacoustic Tomography}

\subsection{1 $\mathrm{Fe}_{3} \mathrm{O}_{4}$ Nanoparticles}

An aqueous suspension of $\mathrm{Fe}_{3} \mathrm{O}_{4}$ nanoparticles was prepared using a typical synthetic method. The nanoparticles had a diameter around $10 \mathrm{~nm}$, were stabilized with citrate and were suspended in water. For comparison, deionized water was used as the control sample in our experiments. The absorption of the $\mathrm{Fe}_{3} \mathrm{O}_{4}$ nanoparticle colloid was measured from 200 $\mathrm{MHz}$ to $20 \mathrm{GHz}$ with an Agilent 8510C vector network analyzer and Agilent 85070E dielectric "dip" probe kit. The absorption of deionized water, the $\mathrm{Fe}_{3} \mathrm{O}_{4}$ nanoparticle colloid and $\mathrm{Fe}_{3} \mathrm{O}_{4}$ nanoparticle colloid with water subtracted is shown in Figure 3. At $3 \mathrm{GHz}$, the absorption of the $\mathrm{Fe}_{3} \mathrm{O}_{4}$ nanoparticle colloid is approximately three times larger than that of deionized water. This indicates that the $\mathrm{Fe}_{3} \mathrm{O}_{4}$ nanoparticles can serve as improved contrast agents with our current TAT system. More importantly, the $\mathrm{Fe}_{3} \mathrm{O}_{4}$ nanoparticle colloid has an absorption that is approximately 70 times greater than that of deionized water at $300 \mathrm{MHz}$. Thus at lower frequencies, the $\mathrm{Fe}_{3} \mathrm{O}_{4}$ nanoparticles can provide significantly increased absorption of microwave energy while the decrease in water absorption provides significantly increased penetration depth by the microwaves. 


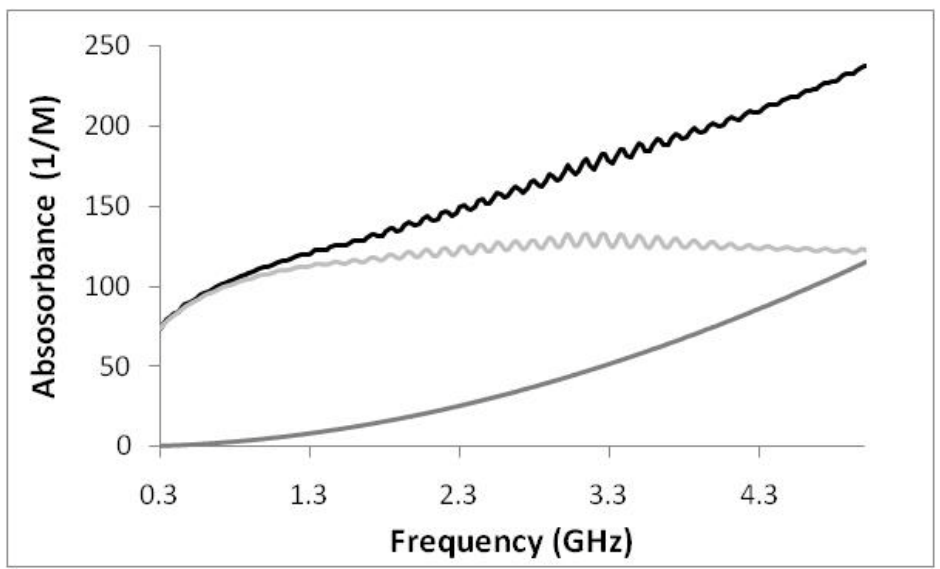

Figure 3: Measured absorbance of the $\mathrm{Fe}_{3} \mathrm{O}_{4}$ nanoparticle colloid (black), deionized water (dark grey) and $\mathrm{Fe}_{3} \mathrm{O}_{4}$ nanoparticle colloid with water subtracted (light grey).

\subsubsection{Thermoacoustic Tomography System}

For the TAT imaging, an imaging system in the Wang lab was used. This microwave excitation source had a center frequency of $3 \mathrm{GHz}$, a pulse width of $0.5 \mu \mathrm{s}$, a peak power estimated to be $2 \mathrm{~kW}$, and a total energy of the microwave pulse estimated to by $1 \mathrm{~mJ}$. The microwave radiation level of this TAT system was estimated to be below the safety requirement. ${ }^{11}$ Due to the modulation frequency, thermoacoustic waves were generated at approximately $1 \mathrm{MHz}$ and are detected by an unfocused transducer (V323, Panametrics Inc.) with a central frequency of $1 \mathrm{MHz}$. The transducer could be fixed to measure a time-resolved thermoacoustic signal or rotated about the sample to form a two dimensional TAT image from the two dimensional projection data.

Plastic tubing containing the $\mathrm{Fe}_{3} \mathrm{O}_{4}$ nanoparticle colloid and deionized water were used to in the meaurements. The inner diameter of the tubing was $1 / 16^{\prime \prime}$. The $\mathrm{Fe}_{3} \mathrm{O}_{4}$ nanoparticle colloid generated more than twice the signal from deionized water when simply measuring the time-resolved thermoacoustic signal from the tubing. Combined with an absorption that is three times greater than the deionized water, this indicates that the $\mathrm{Fe}_{3} \mathrm{O}_{4}$ nanoparticles efficiently transduced the microwave energy to thermoacoustic waves.

To validate the idea of using $\mathrm{Fe}_{3} \mathrm{O}_{4}$ nanoparticles as contrast agents, a tissue phantom was created using two tubes with deionized water and two tubes with the $\mathrm{Fe}_{3} \mathrm{O}_{4}$ nanoparticle colloid. All the four tubes were fixed in a piece of porcine fat and sealed on the top and bottom. Porcine fat was selected because it has low microwave absorbance. The phantom was then immersed in mineral oil for index matching with the microwave source and placed on a sample base on the X-Y plane. Following data collection, the image was reconstructed by using a filtered backprojection method. ${ }^{12}$ Figure 4(a) shows the reconstructed TAT image. The tubes with $\mathrm{Fe}_{3} \mathrm{O}_{4}$ nanoparticle colloid are shown as bright spots marked by white arrows, and the tubes filled with deionized water are denoted by black arrows. In Fig. 4(b), the signal strength from one $\mathrm{Fe}_{3} \mathrm{O}_{4}$ nanoparticle colloid sample and one deionized water sample is shown from the depth marked by two black dashed arrows in Fig 4(a). These results indicate an increased contrast of approximately three times at $3 \mathrm{GHz}$. Again, this is consistent with both the increased microwave absorption and the increased time-resolved thermoacoustic signal generated by the $\mathrm{Fe}_{3} \mathrm{O}_{4}$ nanoparticle colloid. 


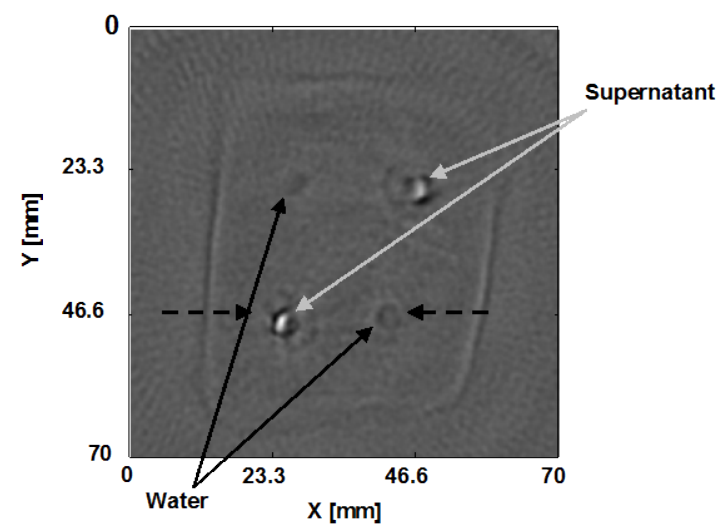

(a)

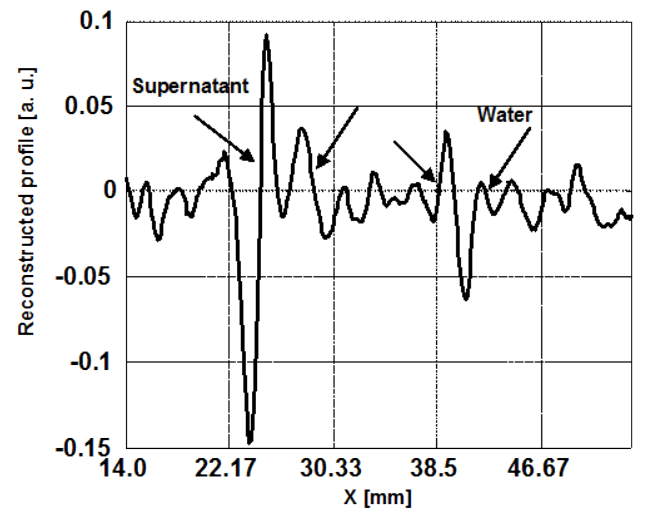

(b)

Figure 4: TAT image (a) Two tubes filled with supernatant sample show as the bright spots in the image, and two tubes filled with deionized water show as the dark circles in the image, (b) Line profile at the depth marked by two black dashed arrows in (a). Figure reprinted from Jin et al. ${ }^{8}$

\section{CONCLUSIONS}

We have presented work covering the use of microwave energy for the synthesis of $\mathrm{ZnO}$ nanoparticles and nanostructures as well as the use of $\mathrm{Fe}_{3} \mathrm{O}_{4}$ nanoparticles as a contrast agent for a hybrid, microwave-based imaging system. The results demonstrate the utility of microwaves in both arenas. In nanoparticle synthesis, microwaves provide unique properties that can impact the synthetic process by delivering energy directly to the solvent or reacting species. This alters the synthetic process and may enable the synthesis of unique structures. In the area of biomedical imaging, the potential of microwaves has only begun to be tapped. Our results indicate that nanoparticle contrast agents may be used with thermoacoustic tomography to allow separation of penetration depth and contrast as currently provided by water. $\mathrm{Fe}_{3} \mathrm{O}_{4}$ nanoparticles represent one possibility for such contrast agents and point to the future development of microwave-based systems at lower frequency in order to improve penetration depth in the body and enable deep tissue imaging.

\section{ACKNOWLEMENTS}

We would like to thank Xing Jin and Aaron Keho for their work on the microwave measurements. RM gratefully acknowledges support of the National Science Foundation.

\section{REFERENCES}

1 Gerbec JA, Magana D, Washington A, Strouse GF, Microwave-enhanced reaction rates for nanoparticle synthesis, J Am Chem Soc 127, 15791-15800 (2005).

2 Gedye R, Smith F, Westaway K, Ali H, Baldisera L, Laberge L, et al., The use of microwave ovens for rapid organic synthesis. Tetrahedron Letters 27, 279-282 (1986). 
3 Giguere RJ, Bray TL, Duncan SM and Majetich G, Application of commercial microwave ovens to organic synthesis, Tetrahedron Letters 27, 4945-4948 (1986).

4 Zhu J, Palchik O, Chen S and Gedanken A, Microwave Assisted Preparation of CdSe, PbSe, and Cu2-xSe Nanoparticles, J. Phys. Chem. B 104, 7344-7347 (2000).

5 Unalan HE, Hiralal P, Rupesinghe N, Dalal S, Milne WI and Amaratunga GAJ, Rapid synthesis of aligned zinc oxide nanowires, Nanotech. 19, 255608 (2008).

6 Wang L, Zhao C, Sun H, and Ku G, Microwave-induced acoustic imaging of biological tissues, Rev. Sci. Instr. 70, 3744-3748 (1999).

7 Kruger R, Stantz KM and Kiser WL, Thermoacoustic CT of the Breast, Proc. SPIE 4682, 521-525 (2002).

8 Jin X, Keho A, Meissner KE and Wang L, "Iron oxide nanoparticles as a contrast agent in thermoacoustic tomography,” Proc. SPIE 6437, 64370E- 1- 7 (2007).

9 Nie L, Ou Z, Yang S and Xing D, Thermoacoustic molecular tomography with magnetic nanoparticle contrast agents for targeted tumor detection, Med. Phys. 37, 4193-4200 (2010).

10 Pramanik M, Swierczewska M, Green D, Sitharaman B and Wang L, Single-walled carbon nanotubes as a multimodalthermoacoustic and photoacoustic-contrast agent, J. Biomed. Opt. 14, 034018 (2009).

11 P. Stavroulakis ed., Biological Effects of Electromagnetic Fields: Mechanisms, Modeling, Biologica Effects, Therapeutic Effects, International Standards, Exposure Criteria, Springer (2003).

$12 \mathrm{Xu} \mathrm{Y}$, Kuchment $\mathrm{P}$ and Wang L, Limited-view thermoacoustic tomography and reconstruction by truncated conjugate gradient, Med. Phys. 31, 724-733 (2004). 